\title{
Oleanolic acid modulates multiple intracellular targets to inhibit colorectal cancer growth
}

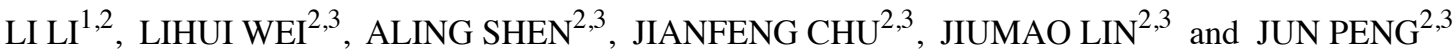 \\ ${ }^{1}$ Department of Disease Prevention and Healthcare, Fujian Provincial Hospital, Fuzhou, Fujian 350001; \\ ${ }^{2}$ Academy of Integrative Medicine and ${ }^{3}$ Fujian Key Laboratory of Integrative Medicine on Geriatric, \\ Fujian University of Traditional Chinese Medicine, Minhou Shangjie, Fuzhou, Fujian 350122, P.R. China
}

Received July 21, 2015; Accepted September 23, 2015

DOI: $10.3892 /$ ijo.2015.3198

\begin{abstract}
Due to drug resistance and unacceptable cytotoxicity of most currently-used cancer chemotherapies, naturally occurring products have gained attention in the field of anticancer treatment. Oleanolic acid (OA) is a natural pentacyclic triterpenoic acid and a principal active compound in many medicinal herbs that have long been used to clinically treat various types of human malignancies. Using a colorectal cancer (CRC) mouse xenograft model and the cell line HT-29, we evaluated the effect of OA on tumor growth in vivo and in vitro, and investigated the underlying molecular mechanisms in the present study. We found that OA significantly inhibited tumor growth in volume and weight in CRC xenograft mice. In addition, OA treatment led to the induction of apoptosis and inhibition of cell proliferation. OA significantly reduced the expression of Bcl-2, Cyclin D1 and CKD4, whereas Bax and p21 expression was profoundly increased after OA treatment. Furthermore, OA significantly suppressed the activation of Akt, p70S6K and MAPK signalings, but promoted p53 pathway activation. Collectively, findings from this study suggest that OA possesses a broad range of anticancer effects via modulation of multiple intracellular targets.
\end{abstract}

\section{Introduction}

Colorectal carcinoma (CRC) is a major global health concern, with over one million new cases and more than half a million

Correspondence to: Dr Jiumao Lin or Dr Jun Peng, Academy of Integrative Medicine, Fujian University of Traditional Chinese Medicine, 1 Qiuyang Road, Minhou Shangjie, Fuzhou, Fujian 350122, P.R. China

E-mail: jiumaolin@hotmail.com

E-mail: pjunlab@hotmail.com

Abbreviations: OA, oleanolic acid; CRC, colorectal cancer; TUNEL, terminal deoxynucleotidyl transferase-mediated dUTP nick end-labeling; PCNA, proliferating cell nuclear antigen; MAPK, mitogen-activated protein kinase

Key words: oleanolic acid, colorectal cancer, signaling pathway, proliferation, apoptosis deaths annually $(1,2)$. The pathogenic mechanisms mediating CRC development are complicated, with involvement of multiple intracellular signaling transduction cascades including mitogen-activated protein kinase (MAPK), serine-threonine kinase Akt, p70S6 kinase (p70S6K) and p53 pathways (3-11). Aberrant activation of these pathways modulates the expression of many key genes, resulting in the imbalance between cell proliferation and apoptosis, and eventually the induction and progression of cancer. Moreover, these pathways usually function redundantly and form a complicated compensatory network via cross-talk. Thus, most currently-used antitumor agents that are designed for single target might not be always effective and their long-term use might generate drug resistance (12-14).

Naturally-occurring products, including traditional Chinese medicines (TCM), have gained increasing attention in the field of anticancer treatment since they contain relatively few side effects as compared to modern chemotherapeutics (15-17). Oleanolic acid (OA), a natural pentacyclic triterpene acid, is an active compound present in many well-known TCM medicinal herbs, such as Hedyotic diffusa, Patrinia scabiosaefolia and Scutellaria barbata. These herbs have long been used in China to clinically treat various types of human malignancies including CRC (18-26). Previous reports suggest OA may contain anti-inflammatory, antioxidant, and antitumor activities (27-29). However, the precise mechanisms of tumorcidal activity of OA remain largely unclear. Using a CRC mouse xenograft model and the cell line HT-29, we evaluated the effect of OA on tumor growth in vivo and in vitro, and investigated the underlying molecular mechanisms.

\section{Materials and methods}

Cell culture. Human colon carcinoma HT-29 cells were obtained from the Cell Bank of Chinese Academy of Sciences (Shanghai, China) and grown in DMEM supplemented with $10 \%$ (v/v) FBS, $100 \mathrm{U} / \mathrm{ml}$ penicillin and $100 \mu \mathrm{g} / \mathrm{ml}$ streptomycin (Invitrogen, Carlsbad, CA, USA). Cells were cultured at $37^{\circ} \mathrm{C}$, in $5 \% \mathrm{CO}_{2}$ humidified incubator.

Oleanolic acid preparation. Oleanolic acid (OA, $\geq 90 \%)$ was purchased from Sigma-Aldrich (St. Louis, MO, USA). For cell-based experiments, stock solution of OA was prepared by 
dissolving OA powder in DMSO to a concentration of $20 \mathrm{mM}$. The working concentrations of OA were made by diluting the stock solution in the culture medium. The final concentrations of DMSO in the medium were $0.1 \%$. For animal experiments, OA was dissolved in saline.

Nude mouse xenograft study. Six-week-old male BALB/c athymic (nude) mice (20-22 g) were obtained from Shanghai SLAC Laboratory Animal Co., Ltd. (Shanghai, China) and housed in the specific pathogen-free controlled conditions $\left(22^{\circ} \mathrm{C}\right.$, a 12 -h light/dark cycle). Food and water were given ad libitum. HT-29 CRC xenograft mice were produced as previously described (19). Briefly, $2.5 \times 10^{6}$ of HT-29 cells were subcutaneously injected into the right flank of mice to initiate tumor growth. At day 3, following xenograft implantation, mice were randomly divided into two groups $(n=10)$ and intraperitoneally injected with $12.5 \mathrm{mg} / \mathrm{kg}$ of OA or saline, daily, 6 days per week for 16 days. Tumor volume was measured and calculated by the formulation of ' $\pi / 6 \times \mathrm{L} \mathrm{x} \mathrm{W}^{2}$ ', where ' $\mathrm{L}$ ' and ' $W$ ' refer to length and width, respectively. All animal experiments were approved by the Institutional Animal Care and Use Committee of Fujian University of Traditional Chinese Medicine.

Immunohistochemistical analysis. Tumor tissues were analyzed by immunohistochemistry staining as described before (19). Briefly, 8 tumors were randomly selected from OA-treatment or control groups. Tumor tissues were fixed in $10 \%$ formaldehyde for $12 \mathrm{~h}$, paraffin-embedded, sectioned and placed on slides. The slides were subjected to antigen retrieval and endogenous peroxidase activity was quenched with hydrogen peroxide. Non-specific binding was blocked with normal serum in PBS (0.1\% Tween-20). Rabbit polyclonal antibody against PCNA at dilution of 1:200 (Santa Cruz Biotechnology, CA, USA) was used to detect the relevant proteins. The binding of the primary antibody was demonstrated with a biotinylated secondary antibody, horseradish peroxidase (HRP)-conjugated streptavidin (Dako) and diaminobenzidine (DAB) as the chromogen. The tissues were counterstained with diluted Harris hematoxylin. After staining, five high-power fields (at magnification of $\mathrm{x} 400$ ) were randomly selected in each slide and the proportion of positive cells in each field was determined using a true color multifunctional cell image analysis management system (Image-Pro Plus, Media Cybernetics, USA). PBS was used to replace the primary antibody as a non-specific control.

MTT assay. HT-29 cells were seeded into 96-well plates at $1.0 \times 10^{4}$ cells per well and treated with various concentrations of OA for $24 \mathrm{~h}$ or $50 \mu \mathrm{M}$ of OA for different time-point. After treatment, MTT assay was performed as previously described (20). Briefly, $10 \mu$ l MTT [ $5 \mathrm{mg} / \mathrm{ml}$ in phosphate-buffered saline (PBS)] was added to each well, and the samples were incubated for an additional $4 \mathrm{~h}$ at $37^{\circ} \mathrm{C}$. The purple-blue MTT formazan precipitate was dissolved in $100 \mu 1 \mathrm{DMSO}$. The cell viability was measured at $570 \mathrm{~nm}$ with a Model ELX800 Microplate Reader (BioTek, VT, USA).

Colony formation assay. HT-29 cells were seeded into 6-well plates at a density of $2 \times 10^{5}$ cells/well and treated with various concentrations of OA for $24 \mathrm{~h}$. The cells were harvested, resuspended in medium and re-seeded into 6-well plates at a density of $1.0 \times 10^{3}$ cells/well. After incubation for 10 days in a $37^{\circ} \mathrm{C}$ humidified incubator with $5 \% \mathrm{CO}_{2}$, colonies were counted by light microscopy.

Cell cycle analysis. HT-29 cells were seeded into 6-well plates at a density of $2 \times 10^{5}$ cells/well and treated with indicated concentrations of OA for $24 \mathrm{~h}$. The cells were harvested, resuspended and adjusted to a concentration of $1 \times 10^{6} \mathrm{cells} / \mathrm{ml}$ and fixed in $70 \%$ ethanol at $4^{\circ} \mathrm{C}$ overnight. The fixed cells were washed twice with cold PBS and incubated for $30 \mathrm{~min}$ with RNase $(8 \mu \mathrm{g} / \mathrm{ml})$ and PI $(10 \mu \mathrm{g} / \mathrm{ml})$. The cell cycle phases were analyzed by flow cytometry (Caliber, Becton-Dickinson, CA, USA) as previously described (22). The fluorescent signal was detected through the FL2 channel and the proportion of DNA in different phases was analyzed using ModfitLT Version 3.0 (Verity Software House, Topsham).

TUNEL assay. Paraffin-embedded sections of tumors (4- $\mu \mathrm{m}$ thick) were analyzed by TUNEL staining with TumorTACS in situ kit (R\&D Systems, Minneapolis, MN, USA) according to the manufacturer's recommended protocol. The proportion of apoptotic cells were recorded as DAB-positive cells (brown stained). Five high-power fields (x400) were randomly selected in each slide and the average proportion of positive cells in each field was counted.

Apoptosis analysis in cells with Annexin V/PI staining. HT-29 cells were seeded into 6-well plates at a density of $2 \times 10^{5}$ cells/well and treated with various concentrations of OA for $24 \mathrm{~h}$. The cells were harvested, resuspended and stained with Annexin V/PI (Becton-Dickinson, CA, USA) according to the manufacturer's instructions followed by flow cytometry analysis. The percentage of cells in early apoptosis was calculated by Annexin V-positivity and PI-negativity and the percentage of cells in late apoptosis was calculated by Annexin V-positivity and PI-positivity.

Western blot analysis. The protein expression was detected by western blot analysis. Protein of tumor tissues or HT-29 cells was extracted using RIPA protein extraction kit (Tiangen Biotech, Beijing, China), respectively. The protein were separated by SDS-PAGE and transferred onto PVDF membranes. The membranes were blocked for $1 \mathrm{~h}$ with $5 \%$ non-fat milk and incubated with primary antibody against Cyclin D1, CDK4, p21, Bcl-2, Bax or $\beta$-actin (all in 1:1,000 dilutions; Cell Signaling Technology, Beverly, MA, USA) overnight at $4^{\circ} \mathrm{C}$. The HRP-conjugated secondary antibodies (1:2,000 dilution) were added. Then the levels of protein expression were detected with enhanced chemiluminescence detection.

Bio-Plex phosphoprotein assay. The phosphorylation of multiple proteins was simultaneously detected by Bio-Plex phosphoprotein assay as described before (22). Briefly, $5 \times 10^{5}$ of HT-29 cells were seeded into $25-\mathrm{cm}^{2}$ flasks with $5 \mathrm{ml}$ medium and treated with $50 \mu \mathrm{M}$ of OA for $24 \mathrm{~h}$. Eight tumors were randomly selected from each group and homogenized. 
A
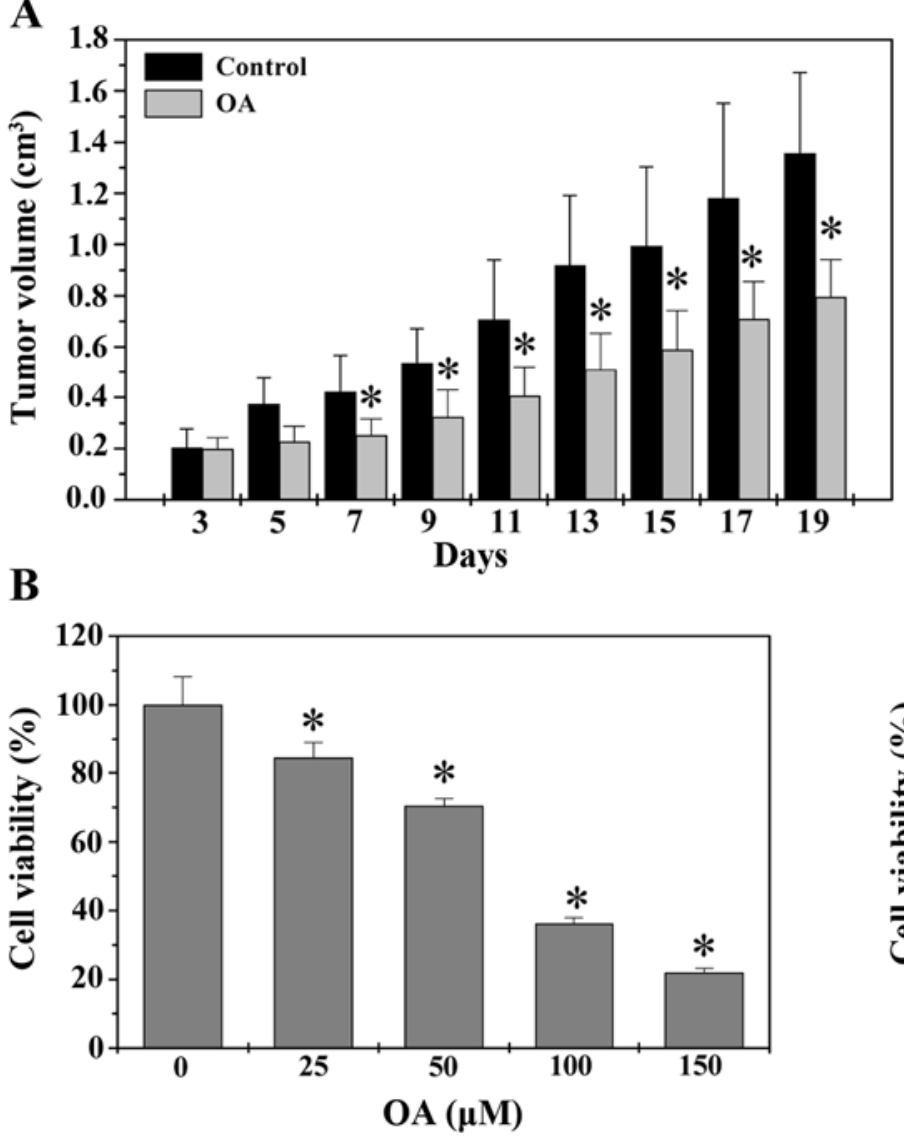
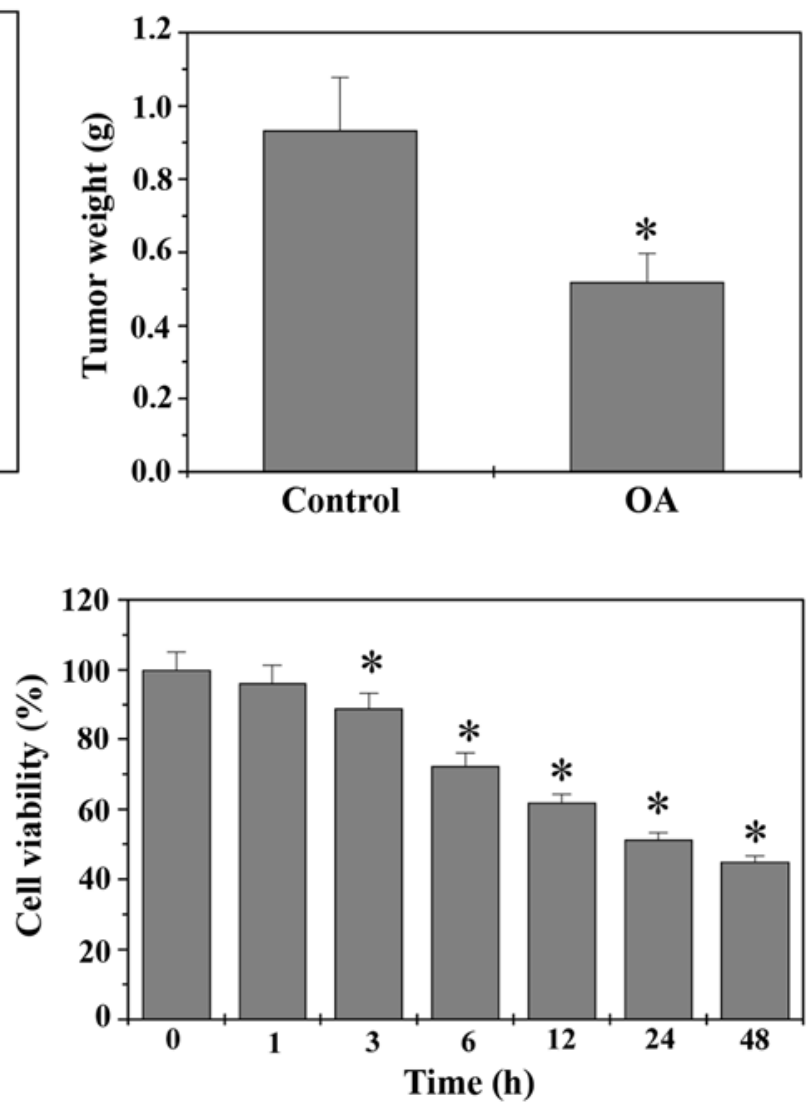

Figure 1. OA inhibits CRC growth. (A) Tumor volume and tumor weight in CRC xenograft mice were measured. Data shown are the averages with SD from 10 mice in OA-treated or control group. (B) HT-29 cell viability was determined by the MTT assay after cells were treated with various concentrations of OA for $24 \mathrm{~h}$ or with $50 \mu \mathrm{M}$ of OA for indicated time periods. Data were normalized to the viability of untreated control cells and shown as the averages with SD from at least three independent experiments. ${ }^{*} \mathrm{P}<0.05$ versus controls.

After lysis of treated cells or tumor tissues the expression of pAkt, pErk1/2, pJNK, pp38, pp70S6K and pp53 was examined using the Bio-Plex 200 suspension array system Bio-Plex Phosphoprotein assay (Bio-Rad, Hercules, CA, USA) according to the manufacturer's protocol.

Statistical analysis. The data were analyzed using SPSS for Windows (Version 17.0) and shown as average with SD. Statistical analysis was performed with Student's t-test or ANOVA.

\section{Results and Discussion}

OA inhibited CRC growth in vivo and in vitro. Tumor growth in vivo was evaluated by measuring the tumor volume and tumor weight in CRC xenograft mice. As shown in Fig. 1A, administration with OA significantly decreased both tumor volume and tumor weight. At the end of experiment, the tumor volume and tumor weight per mouse in control group were $1.36 \pm 0.32 \mathrm{~cm}^{3}$ and $0.94 \pm 0.14 \mathrm{~g}$, whereas those in OA-treated group were $0.79 \pm 0.14 \mathrm{~cm}^{3}$ and $0.51 \pm 0.08 \mathrm{~g}(\mathrm{P}<0.05)$. The in vitro effect of $\mathrm{OA}$ on $\mathrm{CRC}$ growth was determined by MTT assay to compare the relative viability of HT-29 cells in OA-treated monolayers and untreated controls. As shown in Fig. 1B, treatment with $25-150 \mu \mathrm{M}$ of OA for $24 \mathrm{~h}$ reduced
HT-29 cell viability by $16-78 \%$ compared to untreated control cells; and $50 \mu \mathrm{M}$ of OA gradually decreased cell viability with the increase of exposure time $(\mathrm{P}<0.05)$. Taken together, it is suggested that OA possesses inhibitory effects on CRC growth both in vivo and in vitro, in a dose- and time-dependent manner.

OA inhibited CRC cell proliferation through blockage of cell cycle G1 to $S$ progression. Cancers are typically characterized by an uncontrolled increase of cell proliferation (30); which therefore is a critical target of anticancer treatment. To determine the in vivo effect of $\mathrm{OA}$ on cancer cell proliferation, we performed immunohistochemical staining (IHS) to evaluate the expression level of a proliferation marker PCNA in CRC tumor tissues. As shown in Fig. 2A, OA treatment significantly decreased the protein expression of PCNA in CRC xenograft mice. The percentage of PCNA-positive cells in tumors from control and OA-treated CRC mice was $30.64 \pm 5.18$ and $14.75 \pm 5.17 \%$, respectively $(\mathrm{P}<0.05)$. The effect of OA on CRC cell proliferation in vitro was assessed by colony formation assay. As shown in Fig. 2B, OA significantly and dose-dependently decreased the survival rate of HT-29 cells $(\mathrm{P}<0.05)$. Eukaryotic cell proliferation is tightly regulated by the cell cycle, and G1/S transition is a main checkpoint critical for the control of the cell cycle progress. To 

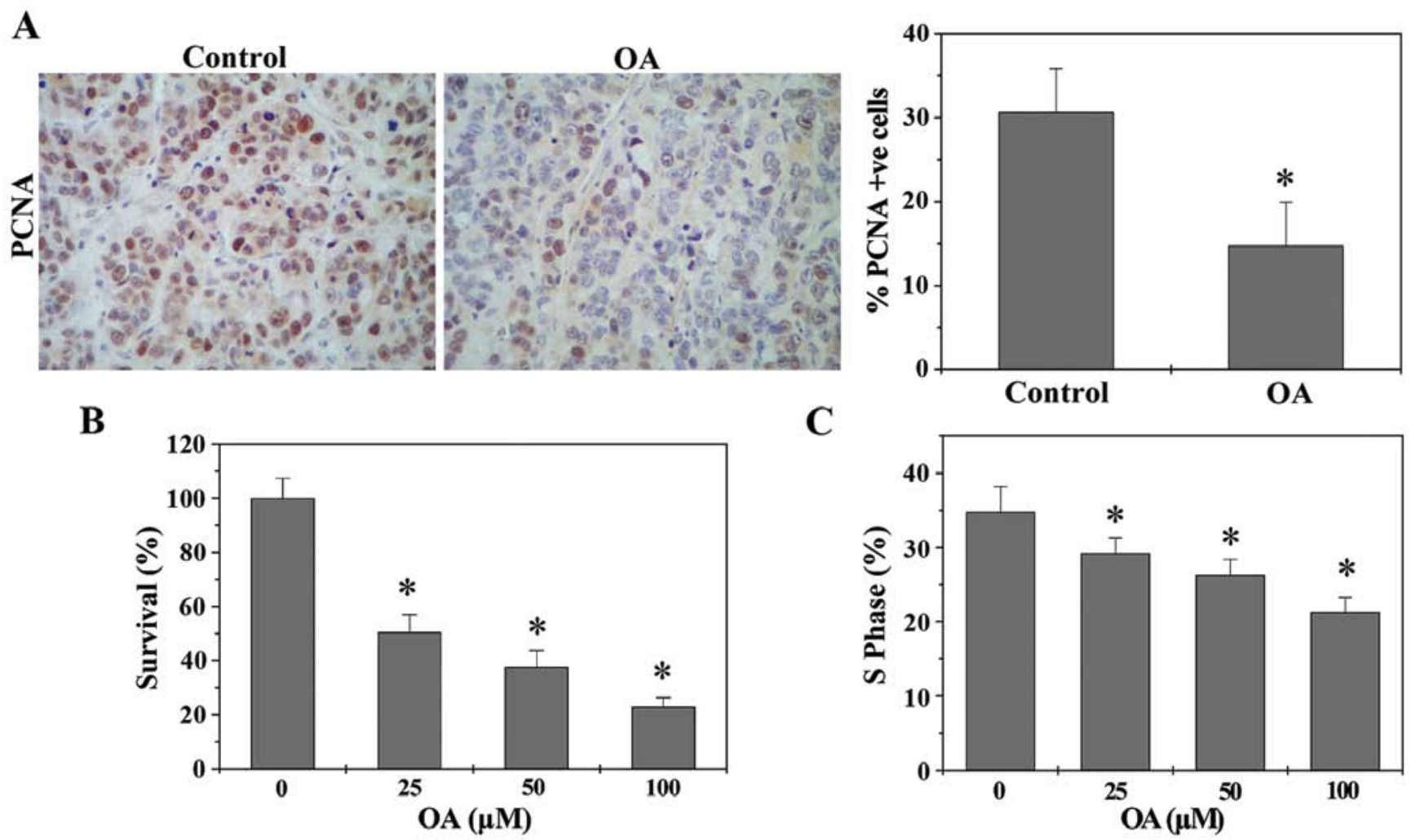

Figure 2. OA inhibits CRC cell proliferation through blockage of cell cycle G1 to S progression. (A) Expression of PCNA in tumor tissues was examined by immunohistochemical staining (IHS). Images are representative taken at a magnification of x400. Quantification of IHS assay is presented as percentage of positively-stained cells and shown as averages with SD from 8 mice in OA-treated or control group. (B) Survival of HT-29 cells was determined using colony formation analysis. Data were normalized to the survival of control cells and shown as the averages with SD from at least three independent experiments. (C) HT-29 cell cycle progression was analyzed by FACS with PI staining. The proportion of cells in S-phase was calculated and shown as the averages with SD from three independent experiments. ${ }^{*} \mathrm{P}<0.05$, versus control cells.

investigate the mechanism of anti-proliferative effect of OA, we performed FACS analysis with PI staining to evaluate cell cycle. As shown in Fig. 2C, OA treatment significantly and dose-dependently reduced the S-phase proportion of HT-29 cells. Collectively, OA can inhibit CRC cell proliferation via $\mathrm{G} 1 / \mathrm{S}$ cell cycle arrest.

OA promoted CRC cell apoptosis via the mitochondriondependent pathway. Apoptosis eliminates abnormal cells and hence is essential for tissue homeostasis. Dysregulation of apoptosis represents a major causative factor of tumorigenesis (31). Therefore, promoting cell apoptosis has been a major focus for antitumor therapies. The in vivo apoptosis was evaluated by TUNEL assay. As shown in Fig. 3A, treatment with OA significantly enhanced the percentage of TUNEL-positive cells in tumors from CRC mice. HT-29 cell apoptosis in vitro was examined using Annexin V/PI staining followed by FACS analysis. As shown in Fig. 3B, OA treatment increased the percentage of cells undergoing apoptosis in a dose-dependent manner.

The mitochondrion-dependent pathway is the most common apoptotic pathway in vertebrate animal cells. Mitochondrial outer membrane permeabilization (MOMP) results in the release of numerous apoptogenic proteins and eventually induces apoptosis, which thereby is a key commitment step in the induction of cellular apoptosis. During the process of
MOMP the electrochemical gradient across the mitochondrial membrane collapses. Therefore, the loss of mitochondrial membrane potential is a hallmark for apoptosis. Since the membrane-permeant JC-1 dye displays potential-dependent accumulation in mitochondria, indicated by a fluorescence emission shift from green $(525 \mathrm{~nm})$ to red $(590 \mathrm{~nm})$, collapse of mitochondrial potential during apoptosis can be represented by a decrease in the ratio of red/green fluorescence intensity of JC-1. As shown in Fig. 3C, OA treatment significantly and dose-dependently reduced JC-1 red/green fluorescent ratio or mitochondrial membrane potential in HT-29 cells $(\mathrm{P}<0.05)$. These data together suggest that OA induces CRC cell apoptosis in vivo and in vitro via the mitochondrion-dependent pathway.

$O A$ regulates the expression of Cyclin D1, CDK4, p21, Bcl-2 and Bax. G1/S progression is highly mediated by Cyclin D1 and its catalytic partner CDK4 $(32,33)$. An unchecked or hyperactivated Cyclin D1/CDK4 complex often leads to uncontrolled increase in cell proliferation (34,35). As a proliferation inhibitor, $\mathrm{p} 21$ protein plays a role in $\mathrm{G} 1$ arrest by binding to and inhibiting the activity of Cyclin-CDK complexes (36). Bcl-2 family proteins are critical mediators of mitochondrion-dependent apoptosis, functioning as either promoters (e.g., Bax) or inhibitors (e.g., Bcl-2) (37). The ratio of Bcl-2 to Bax is critical for determining the fate of cells. 
A

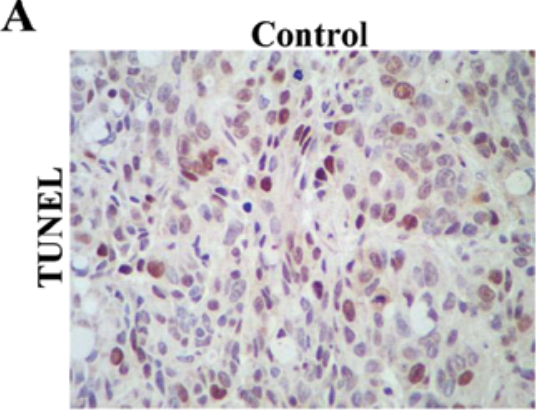

B
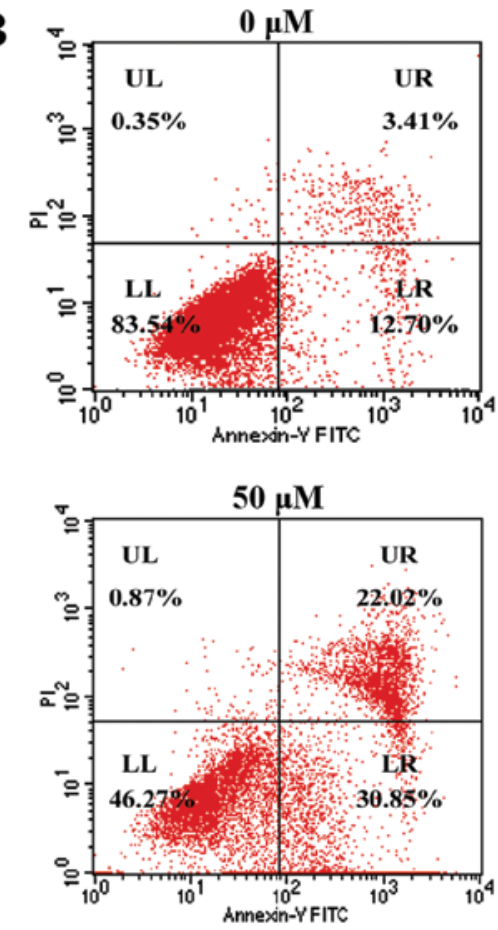

OA

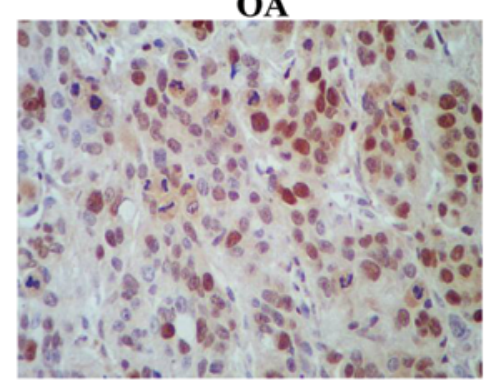

$25 \mu \mathrm{M}$
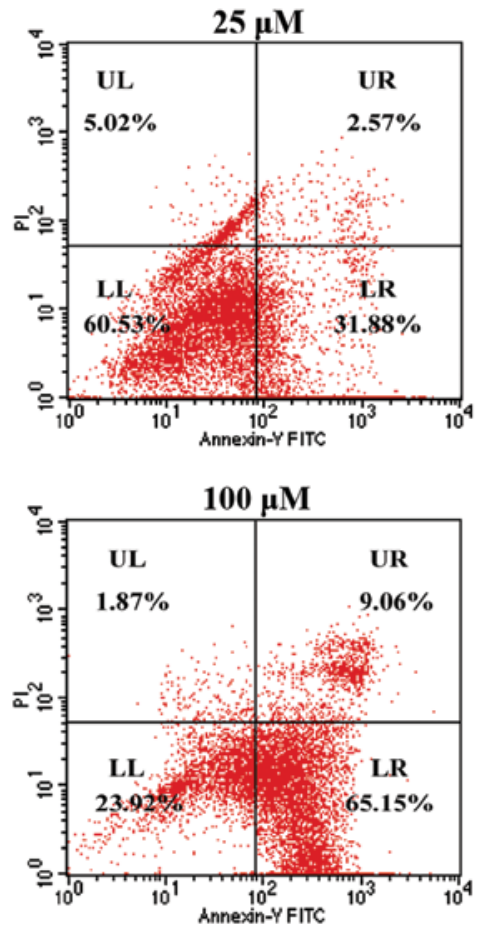
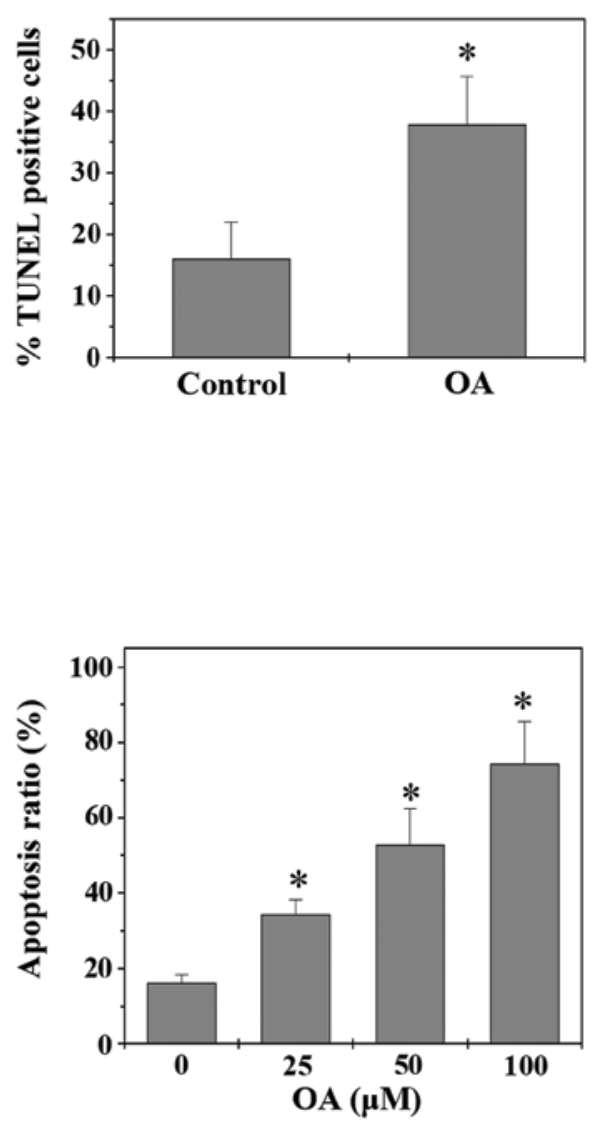

C
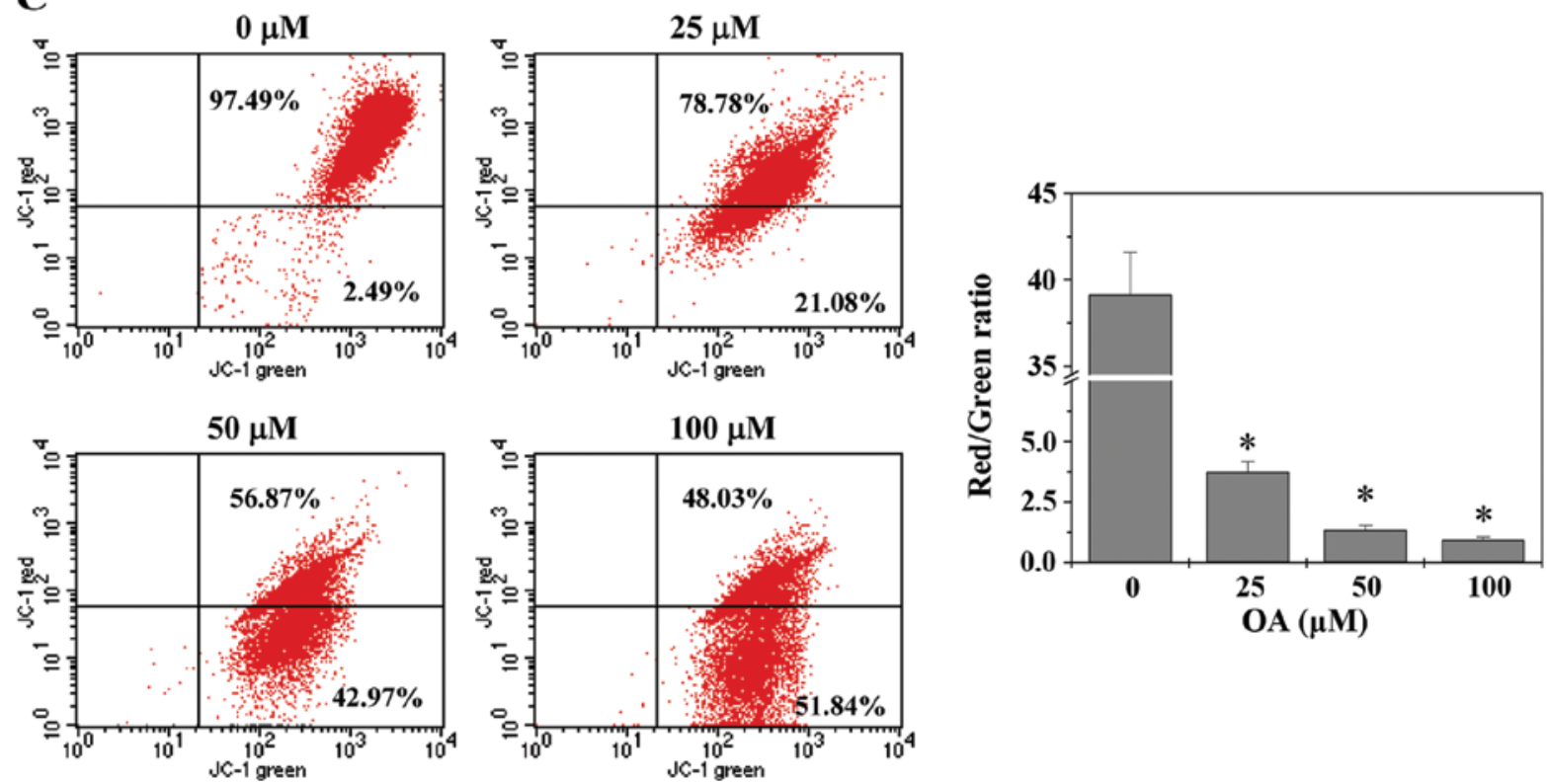

Figure 3. OA promotes CRC cell apoptosis via the mitochondrion-dependent pathway. (A) Apoptosis in tumor tissues was determined by TUNEL. Images are representative taken at a magnification of $x 400$. Quantification of TUNEL assay is presented as percentage of positively-stained cells and shown as the averages with SD from 8 mice in OA-treated or control group. (B) HT-29 cell apoptosis was analyzed by FACS with Annexin V/PI staining. Images are representative of FACS scatter-grams, where Annexin V-positive/PI-negative stained cells (LR, lower right) and Annexin V/PI double-positive stained cells (UR, upper right) represent early apoptosis and late apoptosis, respectively. Quantitative data are shown as the averages with SD from three independent experiments. (C) Mitochondrial membrane potential in HT-29 cells was examined by FACS analysis with JC-1 staining. Images are representative of FACS scatter-grams. Quantitation was calculated as the ratio of red/green fluorescence intensity of JC-1. Data are shown as the averages with SD from three independent experiments. ${ }^{*} \mathrm{P}<0.05$, versus control cells. 
A

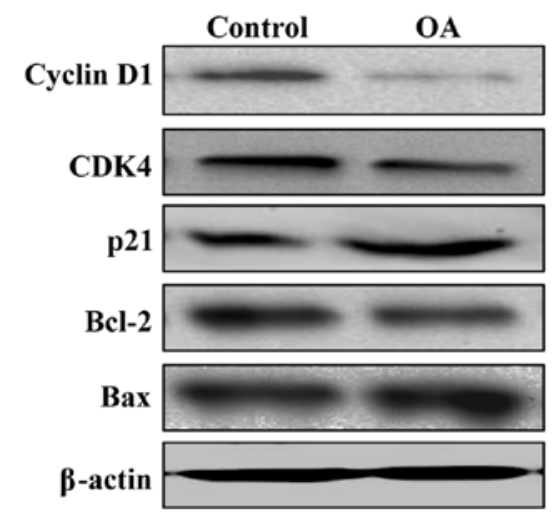

B

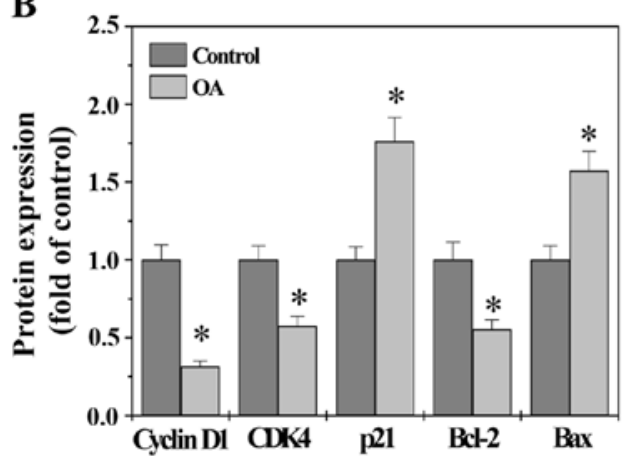

C

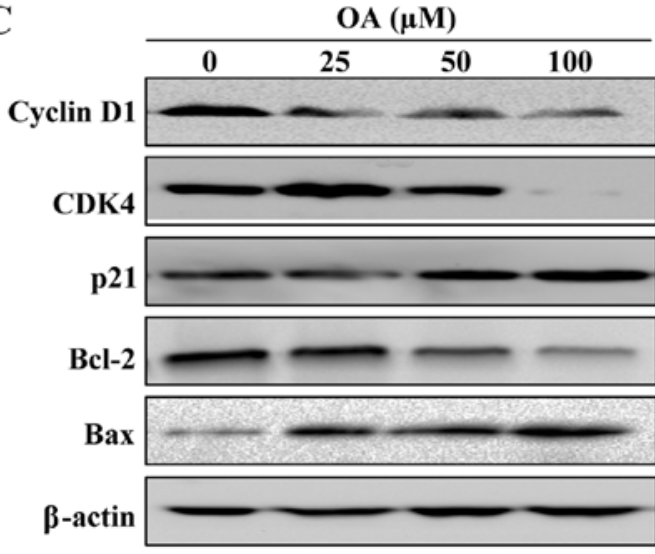

D

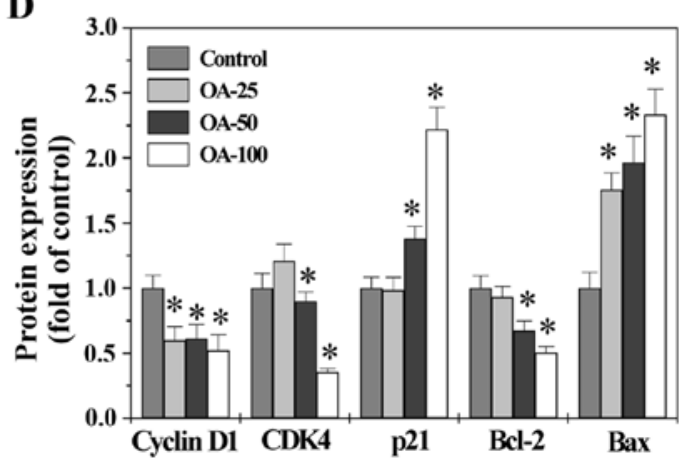

Figure 4. OA regulates the expression of Cyclin D1, CDK4, p21, Bcl-2 and Bax. The protein levels of Cyclin D1, CDK4, p21 Bcl-2 and Bax in tumor tissues (A) or in HT-29 cells (C) were determined by western blot analyses. $\beta$-actin was used as internal control. Images are representative of 3 mice from each animal group or three independent cell-based experiments. (B and D) Densitometric analysis. The data were normalized to the protein expression of untreated controls represented as 'fold of control'. ${ }^{*} \mathrm{P}<0.05$, versus control cells.

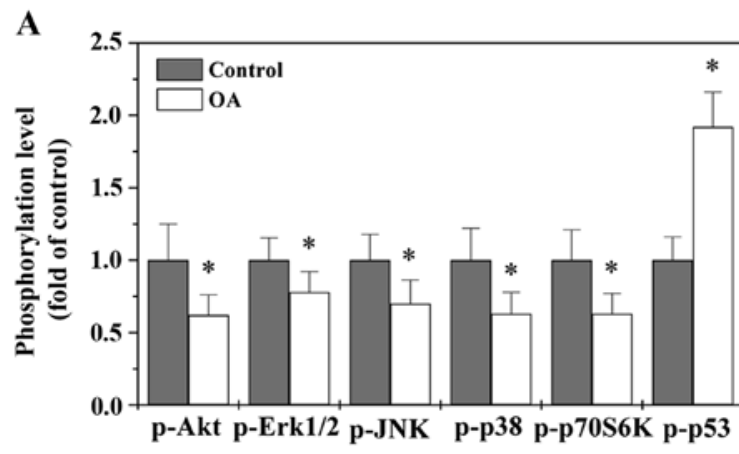

B

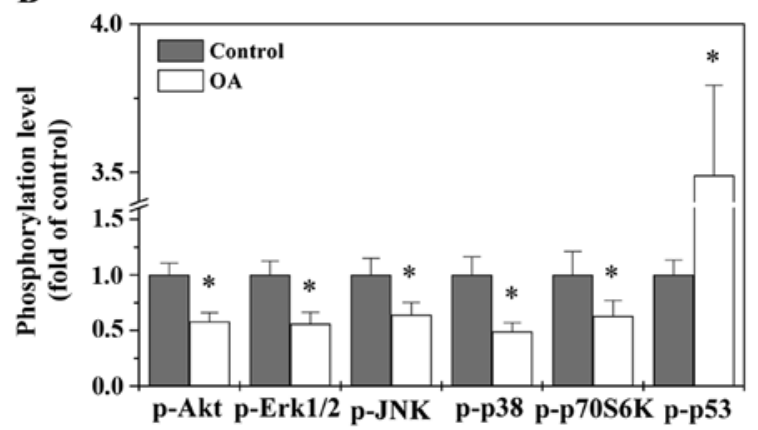

Figure 5. OA modulated the activation of multiple CRC-related signaling pathways. The phosphorylation levels of Akt, p70S6K, p53, Erk1/2, JNK, and p38 in tumor tissues (A) and in HT-29 cells (B) were determined by Bio-Plex Phosphoprotein assay. Data were normalized to the phosphorylation level within controls and represented as fold of control. Data are shown as the averages with SD from 8 mice in each animal group or three independent cell-based experiments. " $\mathrm{P}<0.05$, versus controls.
Higher Bcl-2/Bax ratio is commonly found in various types of cancer, conferring a survival advantage to cancer cells (38-40).

To explore the mechanisms whereby OA exerts its antiproliferative and pro-apoptotic activities, we performed western blot analysis to determine the protein expression of the above-mentioned factors. As shown in Fig. 4, OA significantly reduced the protein level of pro-proliferative Cyclin D1 and CKD4 as well as anti-apoptotic Bcl-2 in both CRC tumors and HT-29 cells, whereas that of anti-proliferative p21 and pro-apoptotic Bax was significantly increased after OA treatment. Taken together, these data demonstrate that OA exerts its antitumor activities through modulating the expression of critical genes involved in cell proliferation and apoptosis.

OA modulates the activation of multiple CRC-related signaling pathways. The development of malignant tumors including $\mathrm{CRC}$ is strongly associated with multiple intracellular signal transduction cascades such as MAPK, Akt, p70S6K and p53 pathways (3-11). After activation by PI3K, Akt phosphorylates mTOR which in turns regulates p70S6K phosphorylation and activation. The Akt-mTOR-p70S6K signaling pathway is considered as a central regulatory pathway of protein expression involved in regulating cell proliferation and survival. Mammals have three major subfamilies of MAPK, including ERK, JNK and p38. The MAPK signaling proceeds via a three-tiered kinase core consisting of MAPK kinase kinase, MAPK kinase, and ultimately a given MAPK. Signaling of both Akt and MAPK are major cell-survival and prolifera- 
tion pathways and have been shown to be activated in several cancers including CRC. In addition, Akt and MAPK pathways can influence the tumor suppressor p53, a transcription factor involved in many cellular processes such as DNA repair, cell cycle arrest and apoptosis. p53 is one of the most frequently mutated genes in human cancers. To further elucidate the mechanisms of antitumor activity of OA, we used Bio-Plex Phospho-protein assay to examine the activation/phosphorylation of multiple proteins in CRC xenograft tumor tissues and HT-29 cells. As shown in Fig. 5, OA treatment significantly reduced the phosphorylation levels of Akt, ERK, JNK, p38 and p70S6K both in vivo and in vitro, while p53 phosphorylation was remarkably increased after OA treatment, suggesting that OA profoundly modulates the activation of multiple CRC-related signaling pathways in vivo and in vitro.

In conclusion, OA exerts its antitumor effects via modulation of multiple cellular targets. Results from this study may provide a strong scientific foundation for the development of novel multi-targeted anticancer agents from the bioactive ingredients in medicinal herbs.

\section{Acknowledgements}

This study was sponsored by the Research Fund for the Doctoral Program of Higher Education of China (20133519110003), the Natural Science Foundation of Fujian Province (2015J01337), and the Developmental Fund of Chen Keji Integrative Medicine (CKJ2014013 and CKJ2015007).

\section{References}

1. Siegel R, Desantis C and Jemal A: Colorectal cancer statistics, 2014. CA Cancer J Clin 64: 104-117, 2014.

2. DeSantis CE, Lin CC, Mariotto AB, Siegel RL, Stein KD, Kramer JL, Alteri R, Robbins AS and Jemal A: Cancer treatment and survivorship statistics, 2014. CA Cancer J Clin 64: 252-271, 2014.

3. Clarke RB: $\mathrm{p} 27^{\mathrm{KIP} 1}$ phosphorylation by PKB/Akt leads to poor breast cancer prognosis. Breast Cancer Res 5: 162-163, 2003.

4. Chang F, Lee JT, Navolanic PM, Steelman LS, Shelton JG, Blalock WL, Franklin RA and McCubrey JA: Involvement of PI3K/Akt pathway in cell cycle progression, apoptosis, and neoplastic transformation: A target for cancer chemotherapy. Leukemia 17: 590-603, 2003.

5. Brunet A, Bonni A, Zigmond MJ, Lin MZ, Juo P, Hu LS, Anderson MJ, Arden KC, Blenis J and Greenberg ME: Akt promotes cell survival by phosphorylating and inhibiting a Forkhead transcription factor. Cell 96: 857-868, 1999.

6. Pratheeshkumar P, Budhraja A, Son YO, Wang X, Zhang Z, Ding S, Wang L, Hitron A, Lee JC, Xu M, et al: Quercetin inhibits angiogenesis mediated human prostate tumor growth by targeting VEGFR-2 regulated AKT/mTOR/P70S6K signaling pathways. PLoS One 7: e47516, 2012.

7. Li W, Tan D, Zhang Z, Liang JJ and Brown RE: Activation of Akt-mTOR-p70S6K pathway in angiogenesis in hepatocellular carcinoma. Oncol Rep 20: 713-719, 2008.

8. Roberts PJ and Der CJ: Targeting the Raf-MEK-ERK mitogenactivated protein kinase cascade for the treatment of cancer. Oncogene 26: 3291-3310, 2007.

9. Schwartsmann G, Di Leone LP, Dal Pizzol F and Roesler R: MAPK pathway activation in colorectal cancer: A therapeutic opportunity for GRP receptor antagonists. Lancet Oncol 6: 444-445, 2005.

10. Fang JY and Richardson BC: The MAPK signalling pathways and colorectal cancer. Lancet Oncol 6: 322-327, 2005.

11. Quartuccio SM, Lantvit DD, Bosland MC and Burdette JE: Conditional inactivation of p53 in mouse ovarian surface epithelium does not alter MIS driven Smad2-dominant negative epithelium-lined inclusion cysts or teratomas. PLoS One 8: e65067, 2013.
12. Van Cutsem E and Costa F: Progress in the adjuvant treatment of colon cancer: Has it influenced clinical practice? JAMA 294: 2758-2760, 2005

13. Longley DB, Allen WL and Johnston PG: Drug resistance, predictive markers and pharmacogenomics in colorectal cancer. Biochim Biophys Acta 1766: 184-196, 2006.

14. Lippman SM: The dilemma and promise of cancer chemoprevention. Nat Clin Pract Oncol 3: 523, 2006.

15. Ma X and Wang Z: Anticancer drug discovery in the future: An evolutionary perspective. Drug Discov Today 14: 1136-1142, 2009.

16. Gordaliza M: Natural products as leads to anticancer drugs. Clin Transl Oncol 9: 767-776, 2007.

17. Newman DJ, Cragg GM and Snader KM: The influence of natural products upon drug discovery. Nat Prod Rep 17: 215-234, 2000.

18. Lin J, Wei L, Shen A, Cai Q, Xu W, Li H, Zhan Y, Hong Z and Peng J: Hedyotis diffusa Willd extract suppresses Sonic hedgehog signaling leading to the inhibition of colorectal cancer angiogenesis. Int J Oncol 42: 651-656, 2013.

19. Cai Q, Lin J, Wei L, Zhang L, Wang L, Zhan Y, Zeng J, Xu W, Shen A, Hong Z, et al: Hedyotis diffusa Willd inhibits colorectal cancer growth in vivo via inhibition of STAT3 signaling pathway. Int J Mol Sci 13: 6117-6128, 2012.

20. Lin J, Chen Y, Wei L, Chen X, Xu W, Hong Z, Sferra TJ and Peng J: Hedyotis Diffusa Willd extract induces apoptosis via activation of the mitochondrion-dependent pathway in human colon carcinoma cells. Int J Oncol 37: 1331-1338, 2010.

21. Lin J, Chen Y, Cai Q, Wei L, Zhan Y, Shen A, Sferra TJ and Peng J: Scutellaria Barbata D Don inhibits colorectal cancer growth via suppression of multiple signaling pathways. Integr Cancer Ther 13: 240-248, 2013.

22. Wei L, Lin J, Wu G, Xu W, Li H, Hong Z and Peng J: Scutellaria barbata D. Don induces G1/S arrest via modulation of p53 and Akt pathways in human colon carcinoma cells. Oncol Rep 29: $1623-1628,2013$

23. Wei L, Lin J, Xu W, Cai Q, Shen A, Hong Z and Peng J: Scutellaria barbata $\mathrm{D}$. Don inhibits tumor angiogenesis via suppression of Hedgehog pathway in a mouse model of colorectal cancer. Int J Mol Sci 13: 9419-9430, 2012

24. Chen L, Liu L, Ye L, Shen A, Chen Y, Sferra TJ and Peng J: Patrinia scabiosaefolia inhibits colorectal cancer growth through suppression of tumor angiogenesis. Oncol Rep 30: 1439-1443, 2013.

25. Liu L, Shen A, Chen Y, Wei L, Lin J, Sferra TJ, Hong Z and Peng J: Patrinia scabiosaefolia induces mitochondrial-dependent apoptosis in a mouse model of colorectal cancer. Oncol Rep 30: 897-903, 2013.

26. Peng J, Chen Y, Lin J, Zhuang Q, Xu W, Hong Z and Sferra TJ: Patrinia scabiosaefolia extract suppresses proliferation and promotes apoptosis by inhibiting the STAT3 pathway in human multiple myeloma cells. Mol Med Rep 4: 313-318, 2011.

27. Liu J: Oleanolic acid and ursolic acid: Research perspectives. J Ethnopharmacol 100: 92-94, 2005.

28. Wang X, Bai H, Zhang X, Liu J, Cao P, Liao N, Zhang W, Wang Z and Hai C: Inhibitory effect of oleanolic acid on hepatocellular carcinoma via ERK-p53-mediated cell cycle arrest and mitochondrial-dependent apoptosis. Carcinogenesis 34: 1323-1330, 2013.

29. Lúcio KA, Rocha GG, Monção-Ribeiro LC, Fernandes J, Takiya CM and Gattass CR: Oleanolic acid initiates apoptosis in non-small cell lung cancer cell lines and reduces metastasis of a B16F10 melanoma model in vivo. PLoS One 6: e28596, 2011.

30. Evan GI and Vousden KH: Proliferation, cell cycle and apoptosis in cancer. Nature 411: 342-348, 2001.

31. Adams JM and Cory S: The Bcl-2 apoptotic switch in cancer development and therapy. Oncogene 26: 1324-1337, 2007.

32. Chen Y, Robles AI, Martinez LA, Liu F, Gimenez-Conti IB and Conti CJ: Expression of G1 cyclins, cyclin-dependent kinases, and cyclin-dependent kinase inhibitors in androgen-induced prostate proliferation in castrated rats. Cell Growth Differ 7: 1571-1578, 1996.

33. Graña X and Reddy EP: Cell cycle control in mammalian cells: Role of cyclins, cyclin dependent kinases (CDKs), growth suppressor genes and cyclin-dependent kinase inhibitors (CKIs). Oncogene 11: 211-219, 1995.

34. Chung DC, Brown SB, Graeme-Cook F, Seto M, Warshaw AL, Jensen RT and Arnold A: Overexpression of cyclin D1 occurs frequently in human pancreatic endocrine tumors. J Clin Endocrinol Metab 85: 4373-4378, 2000 
35. Keum JS, Kong G, Yang SC, Shin DH, Park SS, Lee JH and Lee JD: Cyclin D1 overexpression is an indicator of poor prognosis in resectable non-small cell lung cancer. Br J Cancer 81: 127-132, 1999.

36. Harper JW, Adami GR, Wei N, Keyomarsi K and Elledge SJ: The $\mathrm{p} 21 \mathrm{Cdk}$-interacting protein Cip1 is a potent inhibitor of G1 cyclin-dependent kinases. Cell 75: 805-816, 1993.

37. Adams JM and Cory S: Bcl-2-regulated apoptosis: Mechanism and therapeutic potential. Curr Opin Immunol 19: 488-496, 2007.
38. Peng J, Ding J, Tan C, Baggenstoss B, Zhang Z, Lapolla SM and Lin J: Oligomerization of membrane-bound Bcl-2 is involved in its pore formation induced by tBid. Apoptosis 14: 1145-1153, 2009.

39. Peng J, Tan C, Roberts GJ, Nikolaeva O, Zhang Z, Lapolla SM, Primorac S, Andrews DW and Lin J: tBid elicits a conformational alteration in membrane-bound $\mathrm{Bcl}-2$ such that it inhibits Bax pore formation. J Biol Chem 281: 35802-35811, 2006.

40. Yip KW and Reed JC: Bcl-2 family proteins and cancer. Oncogene 27: 6398-6406, 2008. 